\title{
Correction to: A solution algorithm for integrated production-inventory-routing of perishable goods with transshipment and uncertain demand
}

\author{
Peide Liu' ${ }^{1}$ Ayad Hendalianpour ${ }^{2}\left(\right.$ D $\cdot$ Jafar Razmi $^{3} \cdot$ Mohamad Sadegh Sangari $^{4}$
}

Published online: 16 March 2021

(c) The Author(s) 2021

\section{Correction to: Complex \& Intelligent Systems \\ https://doi.org/10.1007/s40747-020-00264-y}

In the original article, Table 2 was incorrectly published.

The correct table (Table 2) is given below.

The original article has been updated.

The original article can be found online at https://doi.org/10.1007/ s40747-020-00264-y.

Ayad Hendalianpour

a.hendalianpour@soshianest-forecast.com

Peide Liu

peide.liu@gmail.com

Jafar Razmi

jrazmi@ut.ac.ir

Mohamad Sadegh Sangari

mssangari@ut.ac.ir

1 School of Management Science and Engineering, Shandong University of Finance and Economics, Jinan 250014,

Shandong, China

2 Soshianest Enterprise Miner, North Vancouver, BC V7N2J7, Canada

3 School of Industrial Engineering, College of Engineering, University of Tehran, P.O. Box 11155-45632, Tehran, Iran

4 Department of Industrial and Systems Engineering, Fouman Faculty of Engineering, College of Engineering, University of Tehran, P.O. Box 43515-1155, 43516-66456 Fouman, Iran 
Table 2 Comparison between results obtained from the proposed solution algorithm and the ALNS algorithm for the IRPT-ML problem

\begin{tabular}{|c|c|c|c|c|c|c|}
\hline \multirow{2}{*}{ Problem size } & \multirow{2}{*}{ Problem code } & \multicolumn{2}{|c|}{ ALNS algorithm } & \multicolumn{2}{|c|}{ Proposed algorithm } & \multirow{2}{*}{ Improvement (\%) } \\
\hline & & Solution & Time (s) & Solution & Time (s) & \\
\hline \multicolumn{7}{|c|}{ First cluster: Low inventory cost, three time periods } \\
\hline \multirow[t]{3}{*}{ Small } & Acsln 05 & 403.42 & 5.18 & 403.42 & 2.27 & $\mathbf{0}$ \\
\hline & $\operatorname{Acs} \ln 10$ & 1547.29 & 15.37 & 1547.29 & 8.75 & $\mathbf{0}$ \\
\hline & $\operatorname{Acs} \ln 15$ & 1831 & 31.82 & 1830.64 & 16.21 & $\mathbf{0 . 0 2}$ \\
\hline \multirow[t]{4}{*}{ Medium } & $\operatorname{Acs} \ln 20$ & 1999.05 & 52.68 & 1999.05 & 26.88 & $\mathbf{0}$ \\
\hline & Acsln 25 & 2482.82 & 77.15 & 2481.82 & 29.37 & 0.04 \\
\hline & $\operatorname{Acs} \ln 30$ & 3238.33 & 137.53 & 3206.33 & 35.21 & 0.99 \\
\hline & Acsln 35 & 3304.26 & 260.65 & 3252.92 & 14.46 & 1.55 \\
\hline \multirow[t]{3}{*}{ Large } & $\operatorname{Acs} \ln 40$ & 3382.91 & 327.63 & 3446.01 & 35.9 & -1.87 \\
\hline & $\operatorname{Acs} \ln 45$ & 3484.22 & 614.93 & 3224.82 & 21.65 & 7.44 \\
\hline & $\operatorname{Acs} \ln 50$ & 3835.17 & 872.09 & 3666.4 & 30.75 & 4.4 \\
\hline \multicolumn{7}{|c|}{ Second cluster: High inventory cost, three time periods } \\
\hline \multirow[t]{3}{*}{ Small } & Acsln 05 & 1264.68 & 6.12 & 1264.68 & 1.71 & $\mathbf{0}$ \\
\hline & Acsin 10 & 4318.61 & 15.6 & 4316.61 & 4.77 & 0.05 \\
\hline & Acsln 15 & 5279.5 & 33.24 & 5279.5 & 4.79 & $\mathbf{0}$ \\
\hline \multirow[t]{4}{*}{ Medium } & $\operatorname{Acs} \ln 20$ & 6580.57 & 63.63 & 6580.57 & 9.76 & $\mathbf{0}$ \\
\hline & Acs $\ln 25$ & 7842.1 & 79.05 & 7854.16 & 15.26 & -0.15 \\
\hline & Acsln 30 & 11780.3 & 159.95 & 11775.61 & 21.25 & 0.04 \\
\hline & Acsln 35 & 11455.3 & 266.65 & 11384.45 & 22.27 & 0.62 \\
\hline \multirow[t]{3}{*}{ Large } & Acs $\ln 40$ & 13029.9 & 544.99 & 13061.01 & 17.92 & -0.24 \\
\hline & Acs $\ln 45$ & 13604.9 & 587.47 & 13604.42 & 17.01 & $\mathbf{0}$ \\
\hline & Acs $\ln 50$ & 14159.8 & 941.37 & 14088.71 & 31.86 & 0.5 \\
\hline \multicolumn{7}{|c|}{ Third cluster: Low inventory cost, six time periods } \\
\hline \multirow[t]{3}{*}{ Small } & Acsln 05 & 2571.67 & 10.62 & 2571.67 & 6.28 & $\mathbf{0}$ \\
\hline & Acsln 10 & 4244.76 & 40.76 & 4013.96 & 26.07 & 5.44 \\
\hline & Acsln 15 & 4607.52 & 83.09 & 4544.49 & 48.46 & 1.37 \\
\hline \multirow[t]{2}{*}{ Medium } & $\operatorname{Acs} \ln 20$ & 5218.72 & 146.63 & 5218.72 & 58.32 & $\mathbf{0}$ \\
\hline & $\operatorname{Acs} \ln 25$ & 6629.91 & 267.8 & 5892.04 & 62.88 & 11.13 \\
\hline \multirow[t]{2}{*}{ Large } & $\operatorname{Acs} \ln 30$ & 7579.22 & 886.39 & 7526.08 & 51.27 & 0.7 \\
\hline & Acsln 35 & 6801.87 & 633.81 & 6386.02 & 53.29 & 6.11 \\
\hline \multicolumn{7}{|c|}{ Forth cluster: High inventory cost, six time periods } \\
\hline \multirow[t]{2}{*}{ Small } & Acsln 05 & 5112.33 & 12.75 & 5112.33 & 7.07 & $\mathbf{0}$ \\
\hline & Acsln 10 & 8231.27 & 36.9 & 8582.69 & 28.28 & -0.62 \\
\hline \multirow[t]{2}{*}{ Medium } & Acsln 15 & 11366.9 & 90.85 & 11150.34 & 36 & 1.91 \\
\hline & $\operatorname{Acs} \ln 20$ & 13396.6 & 204.31 & 13396.55 & 51.86 & $\mathbf{0}$ \\
\hline \multirow[t]{2}{*}{ Large } & Acs $\ln 25$ & 14821.9 & 332.33 & 14406.9 & 49.37 & 2.8 \\
\hline & Acsln 30 & 23164.5 & 725.36 & 22151.91 & 53.23 & 4.37 \\
\hline
\end{tabular}

Open Access This article is licensed under a Creative Commons Attribution 4.0 International License, which permits use, sharing, adaptation, distribution and reproduction in any medium or format, as long as you give appropriate credit to the original author(s) and the source, provide a link to the Creative Commons licence, and indicate if changes were made. The images or other third party material in this article are included in the article's Creative Commons licence, unless indicated otherwise in a credit line to the material. If material is not included in the article's Creative Commons licence and your intended use is not permitted by statutory regulation or exceeds the permitted use, you will need to obtain permission directly from the copyright holder. To view a copy of this licence, visit http://creativecommons.org/licenses/by/4.0/.

Publisher's Note Springer Nature remains neutral with regard to jurisdictional claims in published maps and institutional affiliations. 